\title{
Novel PRRT2 gene variants identified in paroxysmal kinesigenic dyskinesia and benign familial infantile epilepsy in Chinese families
}

\author{
JIALINZI HE ${ }^{1}$, HAIYUN TANG ${ }^{2}$, CHAORONG LIU ${ }^{1}$, LANGZI TAN ${ }^{1}$, WENBIAO XIAO ${ }^{1}$, \\ BO XIAO ${ }^{1}$, HONGYU LONG $^{1}$ and LILI LONG ${ }^{1}$ \\ Departments of ${ }^{1}$ Neurology and ${ }^{2}$ Radiology, Xiangya Hospital, \\ Central South University, Changsha, Hunan 410008, P.R. China
}

Received July 30, 2020; Accepted February 12, 2021

DOI: $10.3892 /$ etm.2021.9935

\begin{abstract}
The present study was performed to investigate the clinical manifestations and pathogenic variants in three large families with autosomal dominant paroxysmal kinesigenic dyskinesia (PKD) and/or benign familial infantile epilepsy (BFIE) in China. Detailed clinical data and family history were collected. Genomic DNA was isolated from the peripheral blood samples of all available members. The genetic diagnosis was made by whole-exome sequencing on the three probands and the candidate variants were verified by PCR-Sanger sequencing. The pathogenicity of variants was predicted by bioinformatics analyses and classified according to the American College of Medical Genetics criteria. A total of three causative heterozygous variants were identified in the proline-rich transmembrane protein 2 (PRRT2) gene by DNA sequencing: A novel c.324_334del(p.Val109Argfs*21) deletion variant in Family $\mathrm{A}$, as well as the previously known c.510_513del(p.Ser172Argfs*3) deletion variant in Family B and c.649dupC(p.Arg217Profs*8) duplication variant in Family C. The three variants of PRRT2 co-segregated with the phenotype and genotype in the family members. The present results deepen the current understanding of PKD/BFIE and extend the genotypic-phenotypic spectrum of PKD/BFIE.
\end{abstract}

\section{Introduction}

Autosomal dominant neurological disorders include paroxysmal kinesigenic dyskinesia [PKD; Online Mendelian Inheritance in Man (OMIM) 128200] and benign familial infantile epilepsy (BFIE; OMIM 605751), which are related

Correspondence to: Professor Hongyu Long or Professor Lili Long, Department of Neurology, Xiangya Hospital, Central South University, 87 Xiangya Road, Changsha, Hunan 410008, P.R. China

E-mail: longhongyu@cdu.edu.cn

E-mail: longlili1982@126.com

Key words: genotypic-phenotypic spectrum, dystonia, PRRT2, gene variant and may occur either in combination or separately $(1,2)$. PKD is one of the most common hereditary paroxysmal movement disorders. The typical clinical manifestations of PKD are recurrent attacks of choreic and dystonic movements, such as dystonic postures, chorea and athetosis triggered by localized or generalized transient involuntary movements $(3,4)$. The symptoms generally develop in adolescence or early adulthood and last for $<2 \mathrm{~min}$, typically or up to $5 \mathrm{~min}$ on occasion (5). However, usually they spontaneously disappear with age. Anticonvulsant drugs such as carbamazepine and phenytoin may effectively halt the disease's progression and improve the symptoms of patients (6). BFIE is an autosomal dominant epilepsy, which is characterized by focal seizures or may progress to secondarily generalized seizures. The seizures are clustered and have an onset at the age of 3-7 months, and they usually alleviate prior to the age of 2 years with a good prognosis (7). Certain members of families with BFIE may develop PKD during childhood or adolescence $(8,9)$.

It has been reported that gene variants in proline-rich transmembrane protein 2 (PRRT2; NM_001256442.2), potassium voltage-gated channel subfamily A member 1 (KCNA1), cholinergic receptor nicotinic alpha 4 subunit, calcium-activated potassium channel subunit alpha-1 (KCNMA1) and sodium voltage-gated channel alpha subunit 8 (SCN8A) may cause PKD and BFIE. PRRT2 is the dominant pathogenic gene in PKD and BFIE (10-14). The PRRT2 gene is predominantly expressed in the nervous system, particularly in the cerebral cortex, hippocampus, basal ganglia and cerebellum (15). According to the Human Genome Variation Society (HGVS; http://varnomen.hgvs.org/), almost 100 variants have been reported in the PRRT2 gene, including missense, nonsense, frameshift, splice site, deletion and insertion variants.

In the present study, clinical information was collected from three large families with PKD and/or BFIE, and whole-exome sequencing was performed to screen the genetic variants. One novel c.324_334del(p.Val109Argfs*21) frameshift variant and two reported variants c.510_513del(p.Ser172Argfs*3) and c.649dupC(p.Arg217Profs*8) in PRRT2 were identified from these three Chinese families with hereditary PKD/BFIE. In the three families, PRRT2 variants were observed in all family members with PKD/BFIE but not in healthy family members. 


\section{Patients and methods}

Patients. Three families with autosomal dominant disorders were recruited at Central South University Xiangya Hospital (Changsha, China) between May 2018 and August 2019. Clinical information and 4-ml blood samples were collected from three probands and their family members. Written informed consent was obtained from the legal guardians of minors under the age of 18 years and all adult participants and approval was obtained from the Ethics Committee of Xiangya Hospital of Central South University (Changsha, China). The three probands were all diagnosed with PKD for the first time. A detailed analysis of the family history revealed that family B and family $\mathrm{C}$ had a history of BFIE.

Whole-exome sequencing. Genomic DNA was extracted from blood samples using the QIAamp DNA Blood Mini kit (Qiagen $\mathrm{GmbH}$ ) according to the manufacturer's protocol and $0.6 \mu \mathrm{g}$ genomic DNA of each proband was used as input material. Exome capture was performed for each proband using the Agilent SureSelect Human All Exon V6 kit (Agilent Technologies, Inc.) according to the manufacturer's protocol. Using an ultrasonoscope, DNA was sheared into fragments varying from 180 to $280 \mathrm{bp}$ in size. Sequencing-capture DNA libraries were sequenced on the Illumina Hiseq platform (Illumina, Inc.). The original fluorescence image files were transformed to raw data by base calling and these short reads were recorded in FASTQ format, which contained corresponding sequencing quality information. Valid sequencing data were aligned to the human reference genome sequence from the National Center for Biotechnology Information (NCBI) database using the Multi-Vision software package of Burrows Wheeler Aligner (16-17). Small deletions and insertions were determined by the GATK InDel Genotyper and single nucleotide variants were identified using SOAPsnp. Reported variants were filtered through the Single Nucleotide Polymorphism Database (dbSNPs; https://www.ncbi.nlm.nih.gov/projects/SNP/), ExAC browser (http://exac.broadinstitute.org/) and the 1,000 Genomes Project database (www.internationalgenome.org). Previously identified disease-causing mutations were obtained from the Human Gene Mutation Database at the Institute of Medical Genetics in Cardiff (HGMD; http://www.hgmd.cf.ac.uk/ac/index.php).

Validation of variants. In order to verify the DNA variants generated by whole-exome sequencing, the target sites and their flanking sequences were detected by PCR-Sanger DNA sequencing. Genomic DNA reference sequences of candidate gene PRRT2 (NM_001256442.2) were obtained from the University of California Santa Cruz (UCSC) Genome Browser database (http://genome.ucsc.edu/). The PCR primers were designed via online primer 3.0 (http://primer3. ut.ee/) and their sequences and properties are presented in Table I. Sanger sequencing was then performed for all probands and family members. The PCR mixture contained genomic DNA, deoxynucleoside triphosphate, GC buffer I (Sangon Biotech Co., Ltd.), primer, LA Taq DNA polymerase (Sangon Biotech Co., Ltd.) and deionized water (18). The PCR program was as follows: $95^{\circ} \mathrm{C}$ for $3 \mathrm{~min} ; 36$ cycles of $94^{\circ} \mathrm{C}$ for $30 \mathrm{sec}, 58^{\circ} \mathrm{C}$ for $30 \mathrm{sec}$ and $72^{\circ} \mathrm{C}$ for $45 \mathrm{sec}$; and finally, $72^{\circ} \mathrm{C}$ for $8 \mathrm{~min}$. Agarose gel electrophoresis was used to separate PCR products and the QIAquick Gel Extraction kit (Qiagen $\mathrm{GmbH}$ ) was used to purify the target fragment. The sequencing data from the Applied Biosystems 3730x1 DNA Analyzer (Thermo Fisher Scientific, Inc.) were aligned to the human reference sequence by the CodonCode Aligner to confirm the variant (18).

Bioinformatics analysis. The protein sequences of the human PRRT2 (NP_660282.2) and the homologous sequences of different species in FASTA format were obtained from the NCBI protein database. Conservation analysis and sequence alignment were performed by MEGA software (version 7) (19). The pathogenicity of three frameshift variants was predicted by MutationTaster (http://www.mutationtaster.org), and classified according to the American College of Medical Genetics (ACMG) criteria (20). The 1,000 Genomes Project and Exome Aggregation Consortium (Ex AC) are important resources on human genetic variation supporting studies relating genetic variation to health and disease

\section{Results}

Clinical findings. A total of 24 affected members from the three families with PKD/BFIE were collected for the present study, exhibiting extensive clinical heterogeneity (Fig. 1; Table II). Family A was a fourth-generation Chinese pedigree including nine affected cases who were all diagnosed with idiopathic PKD (Fig. 1, Family A). The proband (subject III7) was a 16-year-old female who had started to have episodic dyskinesia attacks with a frequency of three or four times per month at the age of 8 years. The main clinical symptoms included dystonia and choreoathetosis, which only occurred during the daytime and were mostly triggered by sudden movements. The attacks generally lasted for $<60 \mathrm{sec}$. The patient then received carbamazepine monotherapy, which significantly reduced the attacks. The patients in this family exhibited PKD disease alone and the age of seizure onset in this family was 8-13 years (median, 9.4 years). The other patients in family A did not receive any treatment but the symptoms disappeared prior to the age of 17 years. The clinical characteristics of family A are provided in Table II.

A total of 7 patients in family B had BFIE and the proband (subject III3) had both BFIE and PKD. The patients of family B had seizures at about seven months of age and presented with eye deviation to one side with consciousness, lasting about $10 \mathrm{sec}$. The proband of family B had symptoms of binocular roll-up at six months without incentive, and duration was about $1 \mathrm{~min}$. His condition improved after the patient turned 1 years old. The proband exhibited weakness in the legs when he was running at the age of 10 years and had suffered falls when the condition was severe. The patient's right hand unconsciously tremored. The symptoms that were triggered by sudden movements have not improved so far, and he was 46 years old upon symptom evaluation. The probands and other members of family B had similar symptoms. Excluding the proband, they occurred at 6-8 months of age and symptoms disappeared after one year of age. 
Table I.Primers used for amplification of potential mutations of proline-rich transmembrane protein 2 gene,transcript NM_145239.3.

\begin{tabular}{lccccc}
\hline Mutation & $\begin{array}{c}\text { Mutation } \\
\text { location }\end{array}$ & Forward primer (5'-3') & Reverse primer $\left(5^{\prime}-3^{\prime}\right)$ & Tm $\left({ }^{\circ} \mathrm{C}\right)$ & $\begin{array}{r}\text { Product } \\
\text { size }(\mathrm{bp})\end{array}$ \\
\hline c.324_334del & Exon 2 & CGCTGTCTCTGCTATTCCAT & TTTTGAGGGTGGTGAGTGAG & 58 & 676 \\
c.510_513del & Exon 2 & GCTCCAGAAACCACAGAGAC & GGATGGCAAGGATGATGTAG & 58 & 619 \\
c.649dupC & Exon 2 & GCCAAGAAACAGTGTCCAAA & TGCTTAAGAGCTTGGCTACC & 58 & 639 \\
\hline
\end{tabular}

Tm, temperature at which the extinction value rises to half of the maximum extinction value; del, deletion; dup, duplication.
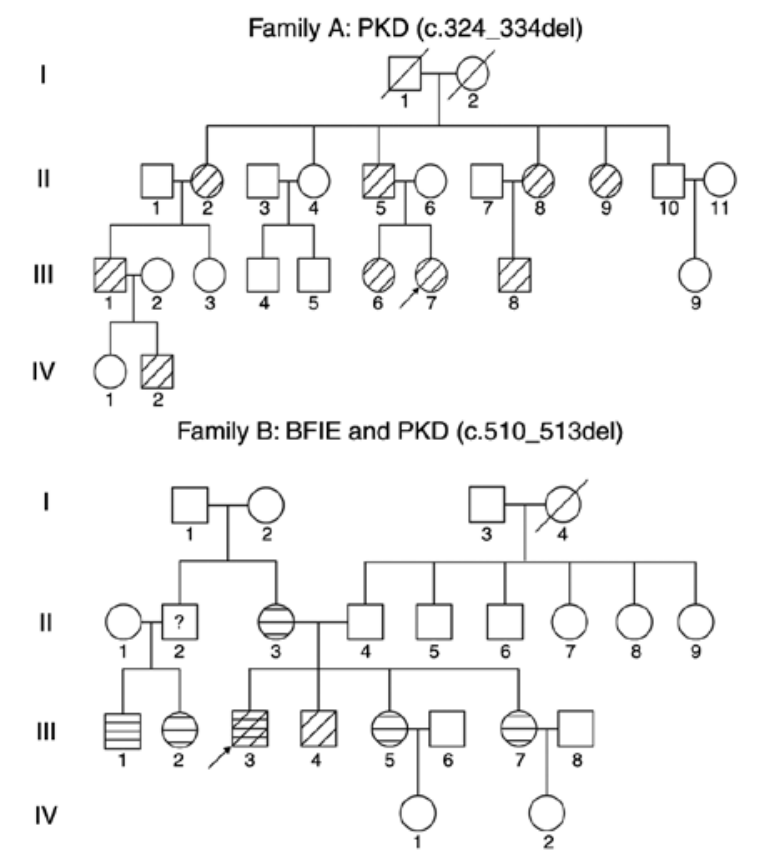

Family C: BFIE and PKD (c.649dupC)
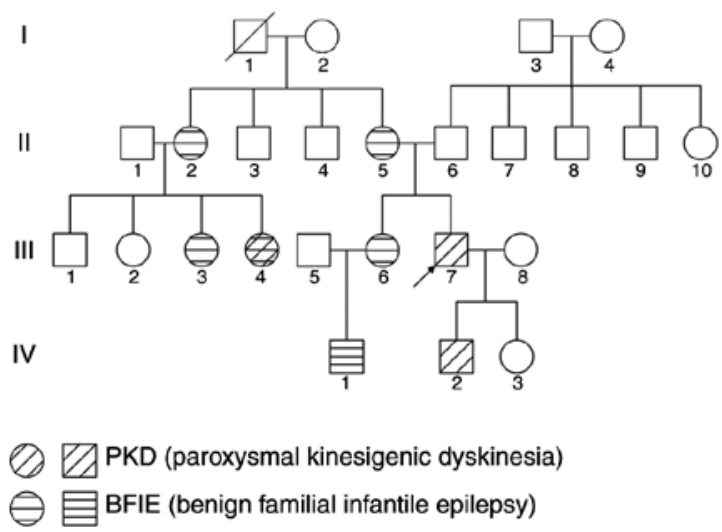

Figure 1. Pedigrees of the three families with PKD/BFIE. Arrows indicate the probands in each family. Question mark indicate deceased individuals who were not examined or tested. PKD, paroxysmal kinesigenic dyskinesia; BFIE, benign familial infantile epilepsy; del, deletion; dup, duplication.

In family $\mathrm{C}$, three patients exhibited $\mathrm{PKD}$, five patients had BFIE and one patient (individual III-4) had both of these diseases. The proband (subject III7) was a 17-year-old male who experienced weakness in both lower limbs when attempting to perform dancing exercise at the age of 15 years. The patient's symptoms improved significantly after taking oxcarbazepine.
Among the affected members of families B and C, 2 had PKD only, 13 had BFIE only and 2 patients had BFIE and PKD combined. The onset age of seizures in the 13 patients with BFIE was 5-8 months (mean age, 6.5 months). Seizures in patients were either resolved spontaneously or with antiepileptic drug treatment. All patients in families B and C had normal results of $24 \mathrm{~h}$ video electroencephalography and brain MRI. The pedigrees of the two families are presented in Fig. 1 and the clinical features of affected individuals were collected and listed in Table II.

Genetic analysis. In order to identify the pathogenic variant responsible for the disease in the three families, whole-exome sequencing was performed on the probands. According to the exome sequencing data, the average coverage was $\sim 99.5 \%$ and the average median depth reached 162 -fold. First, the study focused on identifying deleterious variants in known related genes of paroxysmal movement disorders, mainly related to PKD/BFIE, such as the PRRT2, KCNA1, KCNMA1 and SCN8A genes. The variants that presented with a high frequency in the dbSNP database or the 1000 Genome database were also excluded. A total of three potential pathogenic variants in the PRRT2 gene were confirmed in the three probands associated with PKD/BFIE. They included the novel heterozygous PRRT2 variant c.324_334delAGTGTCCAAAC in pedigree A with a family history of PKD. Furthermore, variant c.510_513del(p. Ser172Argfs*3) in family B and variant c.649dupC(p. Arg217Profs*8) in family $C$ were identified, which had been reported in the previous literature (8).

Bioinformatics analysis of the variants. The PRRT2 variant c.324_334delAGTGTCCAAAC in family A was predicted to produce a truncated protein (p.Val109Argfs*21). The variants co-segregated with the phenotype in each of the three families (Fig. 1) and three variants were further confirmed by Sanger sequencing (Fig. 2). Conservation analysis revealed that the deleted base fragment was conserved in diverse species (Fig. 3). This may corroborate the pathogenicity of the mutant gene fragments. MutationTaster predicted that the three frameshift variants are pathogenic variants. Furthermore, the variant frequencies of c.324_334del and c.510_513del were not recorded in the Exome Aggregation Consortium (ExAC) browser and 1,000 Genomes Project database, indicating that these variants are rare events in the human genome. The ACMG has previously developed guidelines for the interpretation of sequence variation and provided novel criteria for 
Table II. Clinical information of patients in the three families with PKD/BFIE.

A, Family A

\begin{tabular}{|c|c|c|c|c|c|c|c|}
\hline Patient no. & Sex & $\begin{array}{c}\text { Age } \\
\text { (years) }\end{array}$ & $\begin{array}{l}\text { Age at } \\
\text { seizure } \\
\text { onset }\end{array}$ & $\begin{array}{l}\text { Duration of } \\
\text { attacks (sec) }\end{array}$ & $\begin{array}{l}\text { Frequency of } \\
\text { attacks }\end{array}$ & Diagnosis & Symptoms \\
\hline II 2 & Female & 69 & $10 \mathrm{y}$ & 10 & 1 attack/d & PKD & Disappeared after age of 12 years \\
\hline II5 & Male & 63 & $8 \mathrm{y}$ & 5 & 3-4 attacks/m & PKD & Disappeared after age of 16 years \\
\hline II 8 & Female & 58 & $9 y$ & NA & NA & PKD & Disappeared after age of 15 years \\
\hline II9 & Female & 55 & $9 \mathrm{y}$ & NA & 2-3 attacks/w & PKD & Disappeared after age of 13 years \\
\hline III1 & Male & 47 & $10 y$ & NA & 1 attack/d & PKD & Disappeared after age of 15 years \\
\hline III6 & Female & 28 & $13 \mathrm{y}$ & NA & 3-4 attacks/d & PKD & $\begin{array}{l}\text { Were relieved by oral } \\
\text { administration of carbamazepine }\end{array}$ \\
\hline III7 & Female & 16 & $8 \mathrm{y}$ & NA & 3-4 attacks/m & PKD & $\begin{array}{l}\text { Disappeared after age of } 9 \text { years } \\
\text { without treatment }\end{array}$ \\
\hline III8 & Male & 22 & $8 \mathrm{y}$ & NA & 3-4 attacks/w & PKD & Disappeared after age of 13 years \\
\hline IV2 & Male & 16 & $10 \mathrm{y}$ & NA & 3-4 attacks/w & PKD & NA \\
\hline
\end{tabular}

B, Family B

\begin{tabular}{|c|c|c|c|c|c|c|c|}
\hline Patient no. & Sex & $\begin{array}{c}\text { Age } \\
\text { (years) }\end{array}$ & $\begin{array}{c}\text { Age at } \\
\text { seizure } \\
\text { onset } \\
\text { (years) }\end{array}$ & $\begin{array}{l}\text { Duration of } \\
\text { attacks (sec) }\end{array}$ & $\begin{array}{l}\text { Frequency } \\
\text { of attacks }\end{array}$ & Diagnosis & Symptoms \\
\hline II3 & Female & 67 & $6-7 \mathrm{~m}$ & 10 & 1 attack/d & BFIE & $\begin{array}{l}\text { Disappeared after age of } 30 \text { years } \\
\text { without treatment }\end{array}$ \\
\hline III1 & Male & 33 & $7-8 \mathrm{~m}$ & 10 & 2-3 attacks/w & BFIE & Improved after age of 1 year \\
\hline III 2 & Female & 30 & $7-8 \mathrm{~m}$ & 10 & $2-3$ attacks/w & BFIE & Disappeared after age of 1 year \\
\hline III3 & Male & 46 & $6-7 \mathrm{~m}$ & NA & 3-4 attacks/w & PKD/BFIE & Still present \\
\hline III4 & Male & 39 & $8 \mathrm{y}$ & 20 & 4-5 attacks/w & PKD & Improved after age of 1 year \\
\hline III5 & Female & 43 & $6-7 \mathrm{~m}$ & NA & NA & BFIE & NA \\
\hline III7 & Female & 36 & $6-7 \mathrm{~m}$ & 30 & NA & BFIE & NA \\
\hline
\end{tabular}

C, Family C

\begin{tabular}{|c|c|c|c|c|c|c|c|}
\hline Patient no. & Sex & $\begin{array}{c}\text { Age } \\
\text { (years) }\end{array}$ & $\begin{array}{c}\text { Age at } \\
\text { seizure } \\
\text { onset }\end{array}$ & $\begin{array}{l}\text { Duration of } \\
\text { attacks (sec) }\end{array}$ & $\begin{array}{l}\text { Frequency } \\
\text { of attacks }\end{array}$ & Diagnosis & Symptoms \\
\hline II 2 & Female & 56 & $5 \mathrm{~m}$ & 60 & NA & BFIE & Disappeared after age of 13 months \\
\hline II5 & Female & 57 & $6 \mathrm{~m}$ & 30 & NA & BFIE & Disappeared after age of 16 months \\
\hline III3 & Female & 20 & $6 \mathrm{~m}$ & NA & NA & BFIE & NA \\
\hline III4 & Female & 20 & $6 \mathrm{~m}$ & $180-240$ & 5 attacks/d & $\mathrm{PKD} / \mathrm{BFIE}$ & Still present \\
\hline III6 & Female & 41 & $5-6 \mathrm{~m}$ & NA & NA & BFIE & Disappeared after age of 18 months \\
\hline III7 & Male & 42 & $16 \mathrm{y}$ & 240 & NA & PKD & Disappeared after age of 18 years \\
\hline IV1 & Male & 3 & $8 \mathrm{~m}$ & NA & NA & BFIE & Disappeared after age of 19 months \\
\hline IV2 & Male & 17 & $15 \mathrm{y}$ & NA & $10 \mathrm{attacks} / \mathrm{d}$ & PKD & NA \\
\hline
\end{tabular}

Y, year; m, month; w, week; d, day; PKD, paroxysmal kinesigenic dyskinesia; BFIE, benign familial infantile epilepsy; NA, no information available.

classifying pathogenic variants in 2020 (21). According to the ACMG criteria, the variants of family A and family B are likely pathogenic variants and the variant of family $\mathrm{C}$ is a pathogenic variant (Table III). 


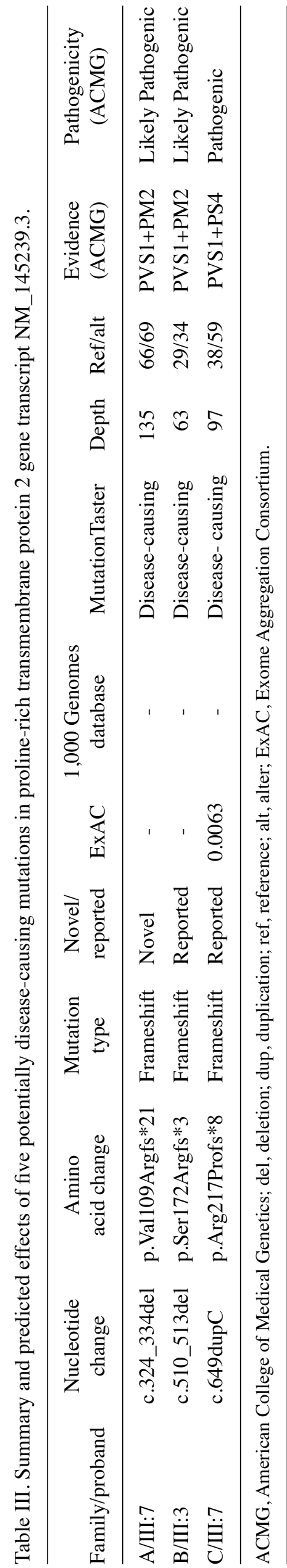

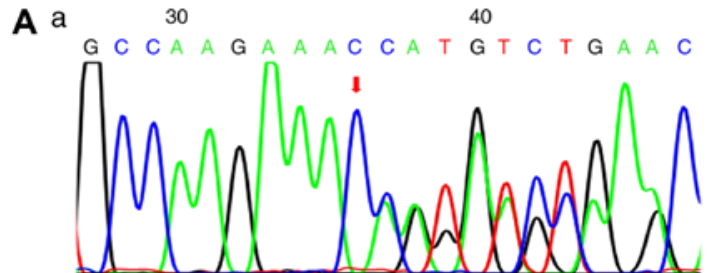

b
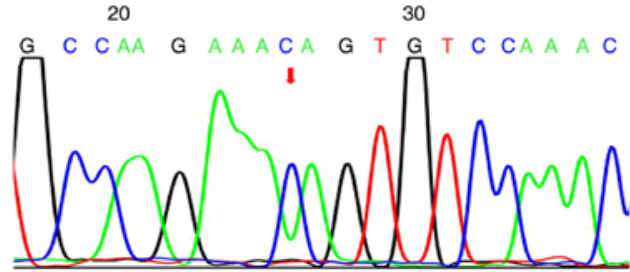

B
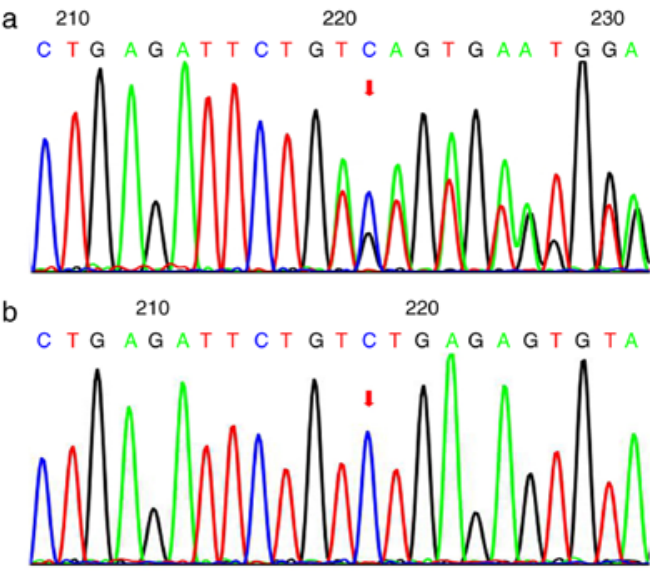

C a

350

360

$G C C C C C C C C C C A A T G G T G C D$

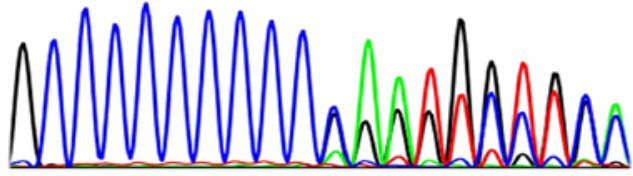

$350 \quad 360$

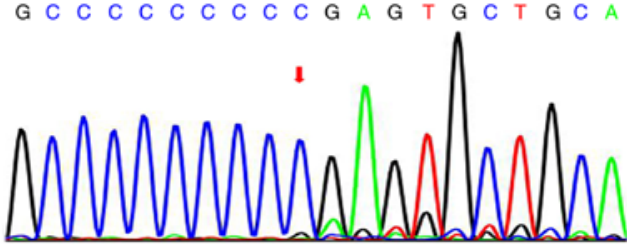

Figure 2. (A) Sequencing chromatograms indicating the PRRT2 variants detected in family A. (a) The mutated sequence and (b) the wild-type sequence. (B) Sequencing chromatograms displaying the PRRT2 variants detected in family B. (a) The mutated sequence and (b) the wild-type sequence. (C) Sequencing chromatograms indicating the PRRT2 variants detected in family C. (a) The mutated sequence and (b) the wild-type sequence. The red arrows indicate the variant site. Position numbers are displayed above the sequences. PRRT2, proline-rich transmembrane protein 2.

\section{Discussion}

Paroxysmal and epilepsy disorders related to involuntary movements have been known for a long time and these disorders may be triggered by several types of stimuli $(22,23)$. PKD is the most common form of paroxysmal movement disorder, which is characterized by recurrent and brief attacks of involuntary movements whose onset may be triggered by sudden 


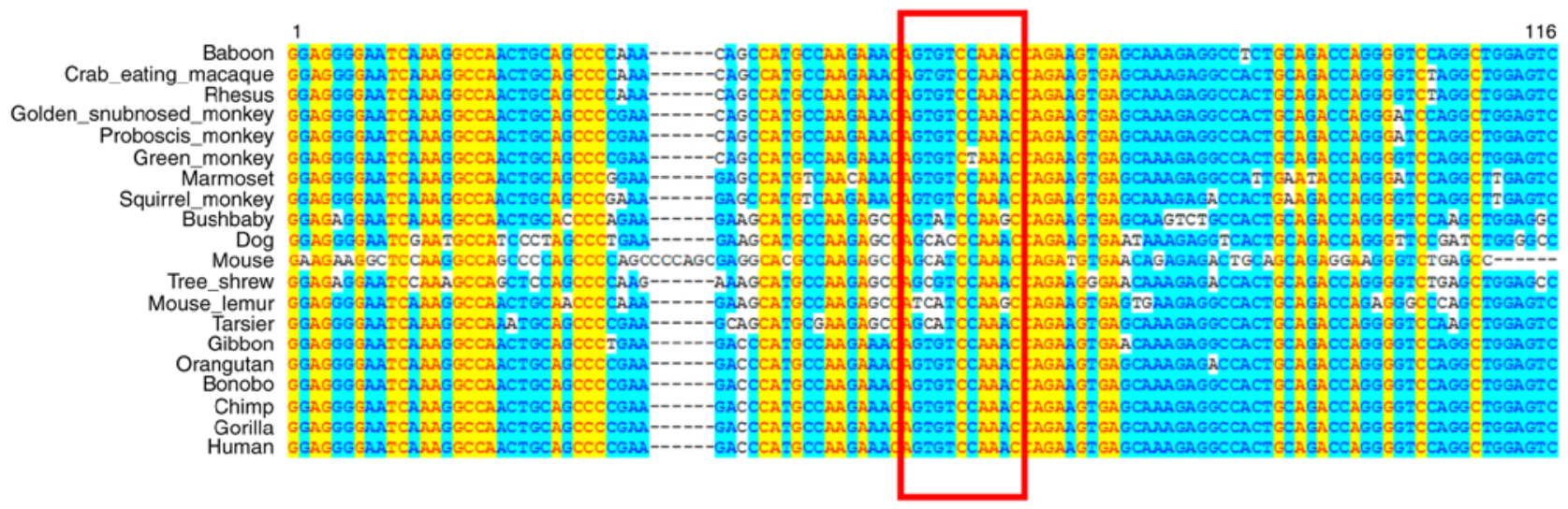

Figure 3. Multiple species sequence alignment indicates the evolutionary conservation of position c.324_334del in proline-rich transmembrane protein 2.

voluntary movements (24). The involuntary movements occur in adolescence or childhood and diminish with age; they usually last several seconds, manifesting as chorea, dystonia or their combination. PKD has a favourable response to relatively low dosages of anticonvulsant medications (25). BFIE is a type of autosomal dominant epilepsy, which begins in infancy (onset between 3 and 12 months of age) and is characterized by brief seizures with motor arrest, hypertonia, cyanosis and limb jerk. The clustered seizures respond well to antiepileptic drugs and usually remit prior to the age of 2 years with a good prognosis (26).

The PRRT2 gene has been reported as a major causative gene for PKD and BFIE. It is also responsible for several familial or sporadic cases with paroxysmal non-kinesigenic dyskinesia, paroxysmal exercise-induced dyskinesia and hemiplegic migraine, but the underlying role of PRRT2 in various pathologies has remained elusive $(27,28)$. The PRRT2 protein is mainly expressed in the central nervous system, regulates the release of synaptic neurotransmitters and facilitates synaptic transmission. To date, $>100$ types of PRRT2 gene variants have been identified. The highest percentage of frameshift variants, which mainly occur in exon 2 , cause unstable truncation proteins of various lengths (29). In the present study, the three frameshift variants identified were predicted to result in the production of a truncation protein and haploinsufficient state. Therefore, the decrease of PRRT2 protein may affect synaptic neurotransmitter release, causing dysregulated neuronal excitability in various brain regions and eventually triggering paroxysmal movement disorders and seizures.

The present study reported on a novel pathogenic PRRT2 variant, c.324_334del, in family A, which was likely responsible for the rare familial neurological disorder PKD. The variant is novel and has not been reported in the 1,000 Genomes Project, dbSNP, Exome Variant Server or ExAC database. Furthermore, all affected cases in the family carried this variant, but it was verified that the healthy individuals did not carry the variant. Evidence of pathogenicity for ACMG's level of evidence of PVS1 should be considered when: The pathogenic mechanism of a disease is loss of function (LOF) or if there are no functional mutations including nonsense, frameshift, classical \pm 1 or 2 splicing, initial codon or single/multiple exon deletion mutations. Furthermore, the following should be considered: i) Whether the LOF of the gene is the clear pathogenic mechanism of the disease; ii) a functional deletion variation at the 3 ' end should be interpreted with caution; iii) attention should be paid to whether the selective deletion of exons affects the integrity of the protein; iv) a gene may have multiple transcripts. Evidence of pathogenicity of ACMG's level of evidence of PM2 should be considered if mutations or low-frequency loci in recessive genetic diseases are not detected in normal controls in the Exome Sequencing Project database, 1,000 Genomes Project database and ExAC database (27). In family A, c.324_334del (p.Val109Argfs*21) was detected as a novel variant, and thus, the evidence was 'PVS1+PM2'. the variants of family A is likely pathogenic variant.

The variant c.510_513del in PRRT2 has been previously reported in one family with PKD (2), and it was detected in family B of the present study, including proband III3 with BFIE, who developed accompanying PKD with age. Chen et al (2) identified a heterozygous 4-bp deletion (514delTCTG) in exon 2 of the PRRT2 gene in the proline-rich domain, resulting in a frameshift and premature termination. The mutation was not present in 1,000 genomes project. Expression of a truncated form of PRRT2 in COS-7 cells led to loss of membrane targeting and localization of the truncated protein in the cytoplasm (28). The symptoms of III3 in pedigree B worsened after taking carbamazepine; however, the symptoms improved after switching to lamotrigine. Li et al (29) reported that low doses of carbamazepine, such as 100 or even $50 \mathrm{mg} /$ day, were able to effectively relieve the symptoms of 12 PRRT2 variant carriers. However, in the present study, carbamazepine was not efficacious for proband (III3) in family B. Thus, the patient required follow-up of the treatment effects for a long time. c.649 is a hotspot variant site: The deletion variant c.649delC, the c.649dupC and a c.649C $>\mathrm{T}$ variant have been reported to affect the same nucleotide $(30,31)$. Among diverse frameshift variants, the c.649dupC frameshift variant is the most common one and may be observed in $80.5 \%$ of cases of PKD. Family $\mathrm{C}$ of the present study with PKD/BFIE carried the hotspot pathogenic variant c.649dupC(p.Arg217Profs*8). While the patients of the family $\mathrm{C}$ carried the same variant, clinical manifestations varied widely across families. For ACMG's level of evidence of PS4, the frequency of the variation in the sick population is significantly higher than that in the control 
population (18). According to the ACMG criteria, the variants of family B are likely pathogenic variant and the variant of family $\mathrm{C}$ is a pathogenic variant.

An optimal diagnosis of PKD and BFIE may be made by combining clinical symptoms, genetic test results and the outcomes of empirical treatment. As the disease was correctly diagnosed, certain patients with PKD were treated with carbamazepine, the most commonly reported anticonvulsant, and patients with BFIE were treated with antiepileptic drugs such as valproate. After a period of treatment, the symptoms of PKD/BFIE may be significantly alleviated (26). Therefore, accurate genetic testing may help doctors make a rapid and precise judgment regarding various paroxysmal diseases and may help patients receive timely treatment with a suitable medicine.

In summary, a genetic analysis in three families with $\mathrm{PKD} / \mathrm{BFIE}$ was conducted and three frameshift variants, including the two deletion variants c.324_334del and c.510_513del and the duplication variant c.649dupC were identified in PRRT2. The present study expanded the variant spectrum of PKD/BFIE, provided further evidence for the precise diagnosis of the disease and may serve as a solid basis for genetic counselling and prenatal gene diagnosis for members of families affected by PKD/BFIE.

\section{Acknowledgements}

Not applicable.

\section{Funding}

This study was supported by the Project from Hunan Provincial Science and Technology Department (grant no. 2019SK1010), the National Natural Science Foundation of China (grant nos. 81671299, 81974206 and 81671300), the Natural Science Foundation of Hunan Province Project (grant no. 2020JJ5914) and the Central South University Graduate Innovation Foundation (grant no. 1053320183945).

\section{Availability of data and materials}

The datasets used and/or analyzed during the current study are available from the corresponding author on reasonable request.

\section{Authors' contributions}

The work was carried out in collaboration between all authors. HL and LL co-designed methods. BX, JH and HT carried out the experiments. CL, LT and WX analyzed the experimental data and performed statistical analysis. JH drafted the manuscript. JH and HT examined the manuscript. HL and LL revised the article. BX and LL confirm the authenticity of all the raw data. All authors have read and approved the final manuscript.

\section{Ethics approval and consent to participate}

All procedures performed in studies involving human participants were in accordance with the ethical standards of the research committee of Xiangya Hospital and with the 1964
Helsinki declaration and its later amendments or comparable ethical standards. Informed consent was obtained from all individual participants or the legal guardians of participants included in the study.

\section{Patient consent for publication}

Written informed consent was obtained from the patients' family for publication of this article and the accompanying images, and the use of data for both the patients and the families.

\section{Competing interests}

The authors declare that they have no competing interests.

\section{References}

1. Lu JG, Bishop J, Cheyette S, Zhulin IB, Guo S, Sobreira N and Brenner SE: A novel PRRT2 pathogenic variant in a family with paroxysmal kinesigenic dyskinesia and benign familial infantile seizures. Cold Spring Harb Mol Case Stud 4: a002287, 2018.

2. Chen WJ, Lin Y, Xiong ZQ, Wei W, Ni W, Tan GH, Guo SL, He J, Chen YF, Zhang Q, et al: Exome sequencing identifies truncating mutations in PRRT2 that cause paroxysmal kinesigenic dyskinesia. Nat Genet 43: 1252-1255, 2011.

3. Bhatia KP: Paroxysmal dyskinesias. Mov Disord 26: 1157-1165, 2011.

4. Albanese A, Bhatia K, Bressman SB, Delong MR, Fahn S, Fung VS, Hallett M, Jankovic J, Jinnah HA, Klein C, et al: Phenomenology and classification of dystonia: A consensus update. Mov Disord 28: 863-873, 2013.

5. van Vliet R, Breedveld G, de Rijk-van Andel J, Brilstra E, Verbeek N, Verschuuren-Bemelmans C, Boon M, Samijn J, Diderich K, van de Laar I, et al: PRRT2 phenotypes and penetrance of paroxysmal kinesigenic dyskinesia and infantile convulsions. Neurology 79: 777-784, 2012.

6. Vigevano F, Fusco L, Di Capua M, Ricci S, Sebastianelli R and Lucchini P: Benign infantile familial convulsions. Eur J Pediatr 151: 608-612, 1992.

7. Okumura A, Hayakawa F, Kato T, Kuno K, Negoro T and Watanabe K: Early recognition of benign partial epilepsy in infancy. Epilepsia 41: 714-717, 2000.

8. Zeng Q, Yang X, Zhang J, Liu A, Yang Z, Liu X, Wu Y, Wu X, Wei $L$ and Zhang Y: Genetic analysis of benign familial epilepsies in the first year of life in a Chinese cohort. J Hum Genet 63: 9-18, 2018.

9. Okumura A, Shimojima K, Kubota T, Abe S, Yamashita S, Imai K, Okanishi T, Enoki H, Fukasawa T, Tanabe T, et al: PRRT2 mutation in Japanese children with benign infantile epilepsy. Brain Dev 35: 641-646, 2013.

10. Jiang YL, Yuan F, Yang Y, Sun XL, Song L and Jiang W: CHRNA4 variant causes paroxysmal kinesigenic dyskinesia and genetic epilepsy with febrile seizures plus? Seizure 56: 88-91, 2018.

11. Usluer S, Kayserili MA, Eken AG, Yiş U, Leu C, Altmüller J, Thiele H, Nürnberg P, Sander T and Çağlayan SH: Association of a synonymous SCN1B variant affecting splicing efficiency with Benign Familial Infantile Epilepsy (BFIE). Eur J Paediatr Neurol 21: 773-782, 2017.

12. Wang J, Gao H, Bao X, Zhang Q, Li J, Wei L, Wu X, Chen Y and Yu S: SCN8A mutations in Chinese patients with early onset epileptic encephalopathy and benign infantile seizures. BMC Med Genet 18: 104, 2017.

13. Yin XM, Lin JH, Cao L, Zhang TM, Zeng S, Zhang KL, Tian WT, $\mathrm{Hu} \mathrm{ZM}$, Li N, Wang JL, et al: Familial paroxysmal kinesigenic dyskinesia is associated with mutations in the KCNA1 gene. Hum Mol Genet 27: 625-637, 2018.

14. Ebrahimi-Fakhari D, Saffari A, Westenberger A and Klein C: The evolving spectrum of PRRT2-associated paroxysmal diseases. Brain 138: 3476-3495, 2015.

15. Rossi P, Sterlini B, Castroflorio E, Marte A, Onofri F, Valtorta F, Maragliano L, Corradi A and Benfenati F: A Novel Topology of Proline-rich transmembrane protein 2 (PRRT2): Hints for an intracellular function at the synapse. J Biol Chem 291: 6111-6123, 2016. 
16. Pruitt KD, Tatusova T and Maglott DR: NCBI Reference Sequences (RefSeq): A curated non-redundant sequence database of genomes, transcripts and proteins. Nucleic Acids Res 33: D501-D504, 2005

17. Li H and Durbin R: Fast and accurate short read alignment with burrows-wheeler transform. Bioinformatics 25: 1754-1760, 2009.

18. Verma M , Kulshrestha S and Puri A: Genome Sequencing. Methods Mol Biol 1525:3-33, 2017.

19. Kumar S, Stecher G and Tamura K: MEGA7: Molecular evolutionary genetics analysis version 7.0 for bigger datasets. Mol Biol Evol 33: 1870-1874, 2016.

20. Richards S, Aziz N, Bale S, Bick D, Das S, Gastier-Foster J, Grody WW, Hegde M, Lyon E, Spector E, et al: Standards and guidelines for the interpretation of sequence variants: A joint consensus recommendation of the American College of Medical Genetics and Genomics and the Association for Molecular Pathology. Genet Meds 17: 405-424, 2015.

21. Riggs ER, Andersen EF, Kantarci S, Kearney H, Patel A, Raca G, Ritter DI, South ST, Thorland EC, Pineda-Alvarez D, et al: Response to Maya et al. Genet Med 22: 1278-1279, 2020.

22. Ma H, Feng S, Deng X, Wang L, Zeng S, Wang C, Ma X, Sun H, Chen R, Du S, et al: A PRRT2 variant in a Chinese family with paroxysmal kinesigenic dyskinesia and benign familial infantile seizures results in loss of interaction with STX1B. Epilepsia 59: $1621-1630,2018$

23. Chen GH: Five cases of paroxysmal kinesigenic dyskinesia by genetic diagnosis. Exp Ther Med 9: 909-912, 2015.

24. Lee HY, Huang Y, Bruneau N, Roll P, Roberson ED, Hermann M, Quinn E, Maas J, Edwards R, Ashizawa T, et al: Mutations in the gene PRRT2 cause paroxysmal kinesigenic dyskinesia with infantile convulsions. Cell Rep 1: 2-12, 2012.

25. Zhao G, Liu X, Zhang Q and Wang K: PRRT2 mutations in a cohort of Chinese families with paroxysmal kinesigenic dyskinesia and genotype-phenotype correlation reanalysis in literatures. Int J Neurosci 128: 751-760, 2018.
26. Heron SE, Grinton BE, Kivity S, Afawi Z, Zuberi SM, Hughes JN, Pridmore C, Hodgson BL, Iona X, Sadleir LG, et al: PRRT2 mutations cause benign familial infantile epilepsy and infantile convulsions with choreoathetosis syndrome. Am J Hum Genet 90: 152-160, 2012.

27. Marini C, Conti V, Mei D, Battaglia D, Lettori D, Losito E, Bruccini G, Tortorella G and Guerrini R: PRRT2 mutations in familial infantile seizures, paroxysmal dyskinesia, and hemiplegic migraine. Neurology 79: 2109-2114, 2012.

28. Ishii A, Yasumoto S, Ihara Y, Inoue T, Fujita T, Nakamura N, Ohfu M, Yamashita Y, Takatsuka H, Taga T, et al: Genetic analysis of PRRT2 for benign infantile epilepsy, infantile convulsions with choreoathetosis syndrome, and benign convulsions with mild gastroenteritis. Brain Dev 35: 524-530, 2013.

29. Li HF, Chen WJ, Ni W, Wang KY, Liu GL, Wang N, Xiong ZQ, $\mathrm{Xu} \mathrm{J}$ and Wu ZY: PRRT2 mutation correlated with phenotype of paroxysmal kinesigenic dyskinesia and drug response. Neurology 80: 1534-1535, 2013.

30. Cao L, Huang XJ, Zheng L, Xiao Q, Wang XJ and Chen SD: Identification of a novel PRRT2 mutation in patients with paroxysmal kinesigenic dyskinesias and c.649dupC as a mutation hot-spot. Parkinsonism Relat Disord 18: 704-706, 2012.

31. Zhang Y, Li L, Chen W, Gan J and Liu ZG: Clinical characteristics and PRRT2 gene mutation analysis of sporadic patients with paroxysmal kinesigenic dyskinesia in China. Clin Neurol Neurosurg 159: 25-28, 2017.

This work is licensed under a Creative Commons Attribution-NonCommercial-NoDerivatives 4.0 International (CC BY-NC-ND 4.0) License. 